\title{
Materials Selection for Ultra-Thin Diamond-Like Carbon Film Metrology and Structural Characterization by TEM.
}

\author{
Guilherme P. Souza ${ }^{1}$, Kurt C. Ruthe ${ }^{1}$, Lifan Chen ${ }^{2}$, Liang Hong ${ }^{2}$ and Haifeng Wang ${ }^{2}$ \\ 1. Western Digital Corporation, Magnetic Heads Operations, Metrology \& Materials Characterization, \\ Ayutthaya, Thailand, 13160 \\ 2. Western Digital Corporation, Magnetic Heads Operations, Materials Characterization, Fremont, CA, \\ USA, 94539
}

Critical dimension metrology by transmission electron microscopy (TEM) plays a paramount role in

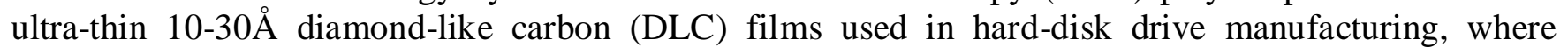
performance is traded against reliability as the nominal thickness continues aggressive scaling downward [1]. TEM sample preparation requires that a protective coating is deposited prior to sitespecific focused ion beam (FIB) cross-sectioning. This coating is also critical in providing a high contrast delineating marker of the DLC top surface for imaging contrast-based metrology. The criteria that such protective coating must be inert to the DLC, as well as free of coarse structure, narrows the materials selection significantly. $\mathrm{Cr}$ is one of the most common coating materials used due to its legacy from SEM coaters for high resolution applications as it forms a continuous, quasi-amorphous film. As the literature on protective layer materials selection for ultra-thin DLC film is non-existent, we disclose original research that has led to a breakthrough in the industry and expanded the current understanding.

DLC film was deposited on NiFe substrate using the conventional filtered cathodic arc (FCA) process [2]. TEM imaging and EELS were performed using a Schottky field emission gun TEM operating at 200 $\mathrm{kV}$, and a post-column spectrometer. Samples, having the DLC film as outer surface layer, were initially coated prior to FIB with either $\mathrm{Cr}$ or $\mathrm{Cr}_{2} \mathrm{O}_{3}$ by ion beam sputtering. $\mathrm{C}$ and $\mathrm{Cr}$ quantified elemental profiles across a Cr-coated DLC film are shown in Fig.1(a). C is clearly skewed towards the $\mathrm{Cr}$ layer, indicating interaction. $\mathrm{C}-\mathrm{K}$ edge extracted from the interfacial region reveals that carbon exists as $(\mathrm{Cr}-)$ carbide, with a characteristically intense $\Pi^{*}$ event [3] (Fig.1b). On the other hand, profile skewness is not present when the same DLC is coated with $\mathrm{Cr}_{2} \mathrm{O}_{3}$ (Fig.1a), and the $\mathrm{C}-\mathrm{K}$ edge does not possess the intense $\Pi^{*}$ indicative of carbide (Fig.1b), retaining a more DLC-like shape instead. Thus the interaction between $\mathrm{C}$ and the protective coating material is central to the interpretation of spectroscopic data.

TEM-based film thickness metrology of the DLC layer is also affected by its interaction with $\mathrm{Cr}$, as shown in Fig.2(a). Using identical film thickness metrology definitions, the $\mathrm{Cr}_{2} \mathrm{O}_{3}$-coated DLC film is $10 \AA$ thicker than the $\mathrm{Cr}$-coated counterpart. The excess film thickness comes from the fact that $\mathrm{C}$ formed carbide with $\mathrm{Cr}$. To evaluate the degree of interaction between DLC and $\mathrm{Cr}_{2} \mathrm{O}_{3}$ (and $\mathrm{Cr}$ ), evaporated $\mathrm{Au}$ film was deposited on the DLC. Evaporated $\mathrm{Au}$ is widely known to be the most gentle and thus the least interacting film deposition technique available. Results show that the Au-coated DLC film thickness is comparable to that of the $\mathrm{Cr}_{2} \mathrm{O}_{3}$-coated film, confirming that $\mathrm{Cr}_{2} \mathrm{O}_{3}$ deposited by the ion beam sputter deposition technique is likewise gentle and non-interacting to the DLC film. However, Au formed large grains (Fig.2b) which disturbed the continuity of its interface with the DLC. Fig.2(c) shows a cross-section of the same DLC sample without any protective coating applied, which was achieved by a very elaborate, manual sample preparation methodology on a Ta coupon substrate that cannot be reproduced for industrial-scale metrology. The uncoated DLC thickness is equivalent to that of evaporated $\mathrm{Au}$-coated and $\mathrm{Cr}_{2} \mathrm{O}_{3}$-coated DLC samples, thus further validating the non-interacting nature 
of $\mathrm{Cr}_{2} \mathrm{O}_{3}$ protective layer for ultra-thin DLC films. Other coating materials tested in this study, such as $\mathrm{Ti}$, Ir, W, and Ta, all show some degree of interaction with $\mathrm{C}$ varying between $\mathrm{Cr}$ and $\mathrm{Cr}_{2} \mathrm{O}_{3}$, and therefore are not as ideal as $\mathrm{Cr}_{2} \mathrm{O}_{3}$ for use as protective marker coating materials for DLC.

In conclusion, ultra-thin DLC characterization by TEM is strongly dependent on the choice of protective material applied prior to cross-sectioning. In particular, the interplay between oxidation and carbidization leads to metrology inaccuracy and misinterpretation of structural information, as well as potential metrology control excursions given that $\mathrm{Cr}$ film stability is dependent on vacuum conditions and target material cleanliness. $\mathrm{Cr}_{2} \mathrm{O}_{3}$ makes a more reliable, stable and robust protective coating system in that regard, allowing for TEM/EELS characterization of the true, artifact-free DLC film at $\AA$ scale.

References:

[1] AC Ferrari, Surf. Coat. Tech. 180-181 (2004), p. 190.

[2] J Robertson, Mater. Sci. Eng. R37 (2002), p. 129.

[3] X Fan et al, Appl. Phys. Lett. 75-18 (1999), p. 2740.

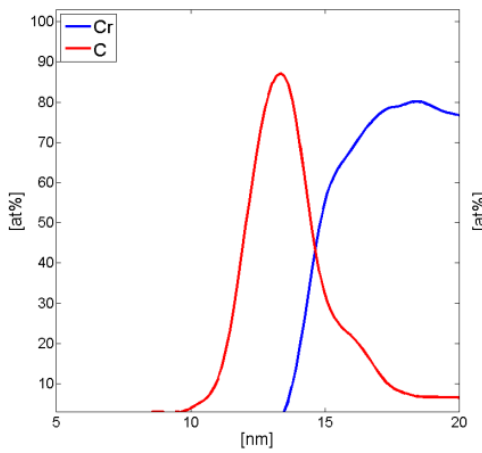

(a)

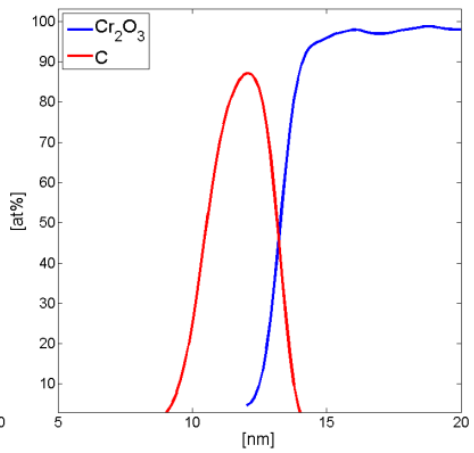

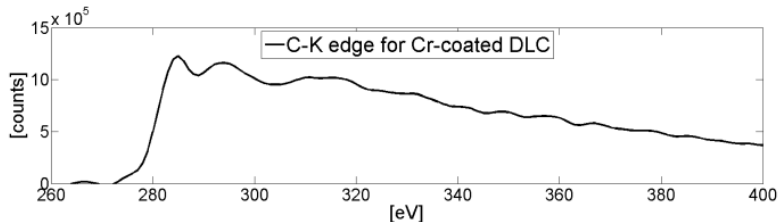

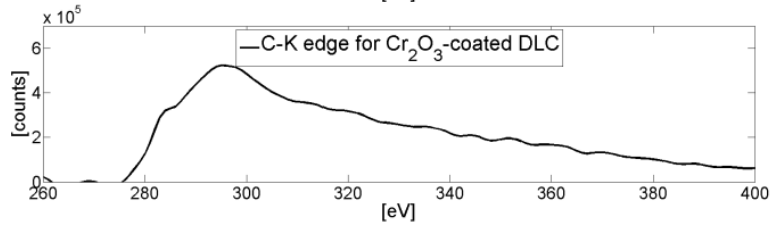

(b)

Figure 1. (a) EELS profiles for $\mathrm{C}, \mathrm{Cr}$, and $\mathrm{Cr}_{2} \mathrm{O}_{3}$ across the DLC. $\mathrm{Cr}$ and $\mathrm{C}$ form a carbide, as shown by the pronounced C-K edge $\Pi^{*}$ event (b) compared to the more DLC-like character retained in the case of the $\mathrm{Cr}_{2} \mathrm{O}_{3}$-coated sample. $\mathrm{C}$-K edges were taken from the interface between DLC and protective layers.

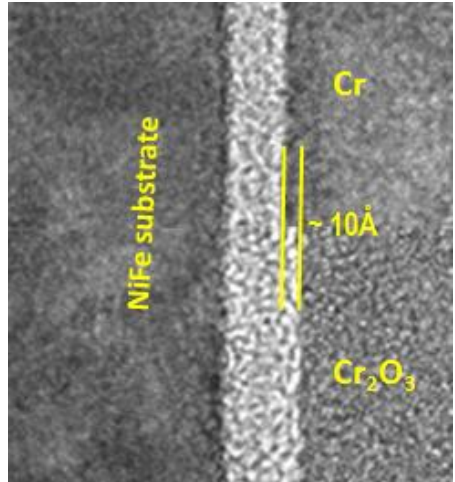

(a)

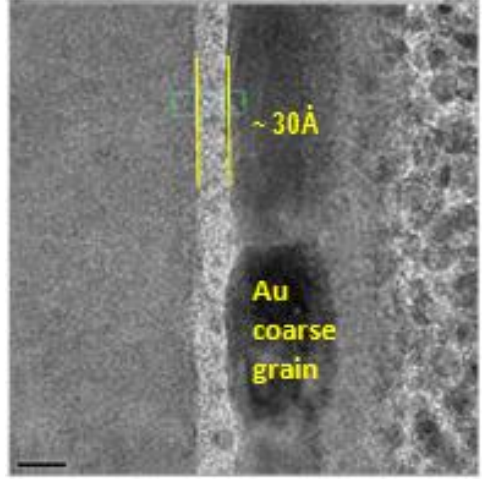

(b)

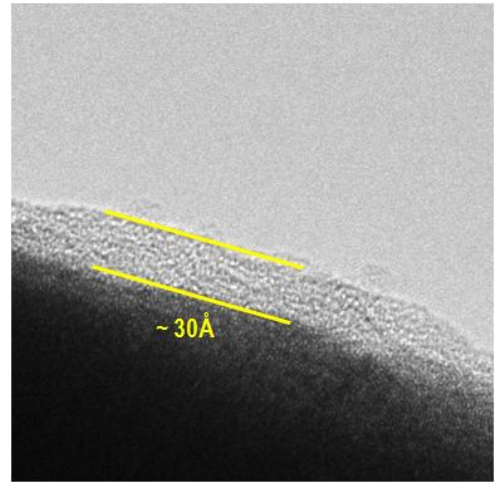

(c)

Figure 2. (a) Comparison between $\mathrm{Cr}$ - and $\mathrm{Cr}_{2} \mathrm{O}_{3}$-coated DLC showing that the $\mathrm{Cr}$-coated DLC is $10 \AA$ thinner due to $\mathrm{C} / \mathrm{Cr}$ interaction; not seen for the $\mathrm{Cr}_{2} \mathrm{O}_{3}$-coated DLC. Evaporated Au-coated (b) and uncoated (c) DLC films have the same thickness as compared to $\mathrm{Cr}_{2} \mathrm{O}_{3}$-coated DLC, confirming that $\mathrm{Cr}_{2} \mathrm{O}_{3}$ protective layer does not interact or change ultra-thin DLC films for TEM characterization. 